\title{
Severe Low Back Pain Due to Retroperitoneal Abscess Formation in Diabetic Patients
}

\author{
Masami Ohmori,Eiji Kobayashi, Kazuhiro Harada, \\ Koh-ichi Sugimoto, Hitoshi Ohkami, Haruhiko Tsutsumi \\ and Akio Fujimura
}

\author{
Department of Clinical Pharmacology and Orhopedics, \\ Jichi Medical School, Tochigi; Advanced Tertiary \\ Emergency Medical Center, Saitama Medical Center, \\ Saitama, Japan
}

\begin{abstract}
We report two cases of diabetic patients with severe low back pain associated with retroperitoneal abscesses. In the first case, multiple retroperitoneal and spinal epidural abscesses were detected. Paraplegia due to the spinal epidural abscess was not relieved by drainage of the abscess and subsequent antibiotic therapy. In the second case, drainage of the retroperitoneal abscess and antibiotics were immediately started, resulting in successful recovery. Thus, we suggest that if a diabetic patient complains of low back pain, potential abscess formations should be considered and given appropriate treatment before administering epidural anesthetic injections for pain relief.
\end{abstract}

Key Words: Low back pain, retroperitoneal abscess, paraplegia, diabetes mellitus.
Patients with diabetes mellitus have a high risk of infection, particularly in a poorly controlled situation [1-3]. It is very difficult to diagnose spinal abscess formation, a life-threatening condition, because of a lack of specific symptoms and signs [4-8]. An adequate diagnosis of paraplegia due to spinal epidural abscess is rarely made [4]. We describe the clinical course of two diabetic patients who had retroperitoneal abscesses with and without spinal epidural abscesses, and review the literature concerning abscesses, especially in the spinal epidural region.

\section{First Case}

A 71-year-old diabetic man with severe lumbago and high fever spikes, was initially admitted to another

Received on 31 March 2002; revised 05 December 2002.

Address for correspondence: Dr. Masami Ohmori, MD, Ph.D. Department of Clinical Pharmacology. Jichi Medical School, Tochigi 329-0498, Japan. Phone: +81-285-58-7388. Fax: +81285-44-7562.E-mail:masami@jichi.ac.jp

The Brazilian Journal of Infectious Diseases 2002;6(6):309-312 (C) 2002 by The Brazilian Journal of Infectious Diseases and Contexto Publishing. All rights reserved.

$1413-8670$ hospital. Although he had been taking glibenclamide (an oral hypoglycemic agent) for the treatment of diabetes mellitus, his control of plasma glucose had been poor for approximately 10 years. After admission, he was treated with several epidural anesthetic injections. The patient was transferred to our hospital to treat the pain symptoms and fever. On admission, he was alert, and his temperature was $36.6^{\circ} \mathrm{C}$, blood pressure 142/ $82 \mathrm{mmHg}$, and pulse rate 100 beats per minute. Physical examination revealed a soft and slightly distended abdomen. Neurological investigation showed reduced vibration sense in both lower legs. No infiltrative shadow was detected on chest and abdominal X-ray examination. His consciousness level acutely decreased to Glasgow Coma Scale 5 on the 5th day after admission. A CT scan of the abdomen was performed to determine the origin of the fever; retroperitoneal abscess formations were detected (Figure 1). We punctured and drained the biggest focus, and found methicillin-sensitive Staphylococcus aureus (MSSA), which was also isolated from the blood and urine. Loss of consciousness was thought to be due to diabetic coma and sepsis. Intensive treatment was initiated with intravenous piperacillin ( $4 \mathrm{~g} / 24$ hours) for infection and with a drip insulin infusion to reduce the 
highblood glucose. The level of consciousness gradually improved, but paraplegia at the $\mathrm{Th}_{4}$ level became evident on the 15th day. As a space-occupying lesion in the vertebral column was suspected, an emergency thoracic MRI scan was done. This revealed an epidural abscess formation that extended below $\mathrm{Th}_{3-4}$ (Figure $2)$. Treatment with the antibiotics, minocycline hydrochloride, aspoxicillin and ciprofloxacin hydrochloride was continued for the next three months. The abscess disappeared and laboratory test results improved after the initiation of glibenclamide $(1.25 \mathrm{mg} /$ day) on the 97th day. However, his paraplegia did not improve. The patient was then transferred to another hospital for ongoing rehabilitation and care.

\section{Second Case}

A 78-year-old diabetic woman with severe low back pain and high fever was admitted to another hospital. Although she had been suffering from diabetes mellitus for seven years, she had taken no medication for this condition. The laboratory data on admission were as follows: WBC, $11.7 \times 10^{3} / \mu$ with $76 \%$ neutrophils; CRP, $17.0 \mathrm{mg} / \mathrm{dl}$; fasting plasma glucose, $225 \mathrm{mg} / \mathrm{dl}$; and hemoglobin ${ }_{\mathrm{AlC}}, 8.9 \%$. To determine if there was a retroperitoneal abscess at the site of low back pain, we immediately performed an abdominal CT, which showed right retroperitoneal abscess formation (Figure 3 ). Drainage of the abscess and antibiotics were initiated. MSSA was isolated in the abscess and urine. Low back pain was relieved, and the abscess disappeared. Laboratory data returned to the normal level on the $50^{\text {th }}$ day.

\section{Discussion}

Diabetic patients are vulnerable to bacterial infections. Many mechanisms have been suggested; the functions (superoxide anion production, chemotaxis, phagocytosis and killing) of neutrophils and monocytes/ macrophages involved in anti-bacterial activity may be reduced in patients with diabetes mellitus [9]. Since antibiotics such as ciprofloxacin increase superoxide anion production by neutrophils [10], such drugs may have merit for diabetic patients with infection. At the same time, blood glucose levels should be precisely controlled in such patients.

Spinal epidural abscess is a very rare disease, but it has an extremely poor prognosis due to the difficulty in diagnosis [4-8]. In the first case, severe low back pain and high fever led us to suspect such an abscess formation. An abdominal CT scan showed multiple abscesses in the retroperitoneal space. We first detected spinal epidural abscesses, with paraplegia, after recovery from loss of consciousness. Intravenous injection of antibiotics, continuous infusion of a fastacting insulin and drainage from a giant retroperitoneal abscess were initiated. The abscess formation and laboratory data improved whereas paraplegia did not change. Based on the experience of the first case, we immediately detected by abdominal CT examination a similar problem in the second case. Prompt drainage of the focus and antibiotics prevented her from developing excessive damage, including an epidural abscess and subsequent paraplegia.

Spinal epidural abscess is characterized as follows: 1) Source of infection: unknown>surgery or other procedures $>$ trauma; 2) Contributing factors: diabetes mellitus, osteomyelitis and others; 3) Clinical symptoms: spinal pain, paralysis and others; 4) Clinical signs: elevations of ESR and WBC, and fever; 5) Most common causative organism: Staphylococcus aureus; 6) Most sensitive procedure for the diagnosis of abscess formation: MRI scan [48]. In the two cases that we examined, urinary tract infection may have been a source of infection as MSSA was detected only in urine and in the retroperitoneal abscess. Although patients with lumbago often receive epidural anesthetic injections, we think that epidural injection is a contributing factor for retroperitoneal abscesses.

When an abscess is detected, drainage is the firstline of treatment. As abdominal CT revealed multiple retroperitoneal abscesses in the first case, drainage of the abscess was done and MSSA was detected in the lesion. Antibiotics showing an in vitro efficacy against the bacteria, along with fast-acting insulin, were intravenously administered and there was a gradual 
Figure 1. Computed transitional tomography scan of the pelvis in the first case. Abscess formations with a gas component are obvious mainly on the left side of the retroperitoneal space.

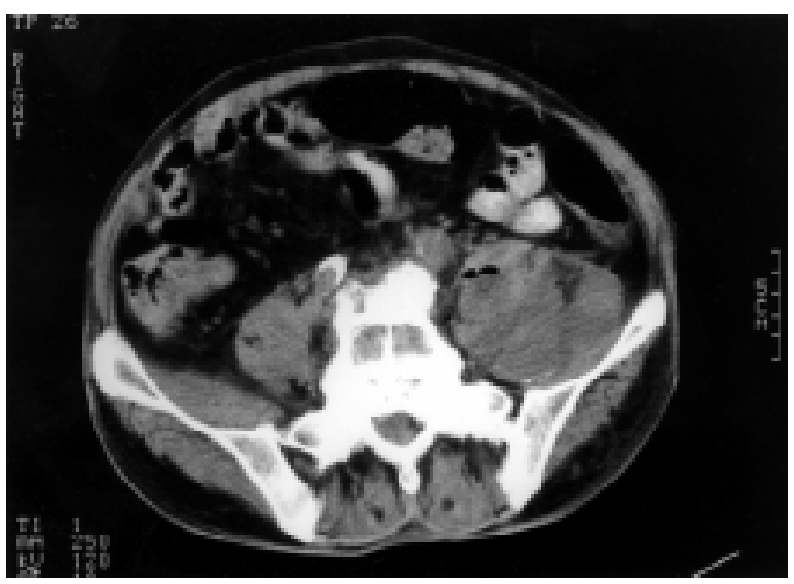

Figure 2. Saggital $\mathrm{T}_{2}$-weighted magnetic resonance imaging scan with gadolinium enhancement of the thoracic spine in the first case. Epidural abscess is evident below the $\mathrm{Th}_{3-4}$ level, and paraspinal abscess and osteomyelitis were also detected.

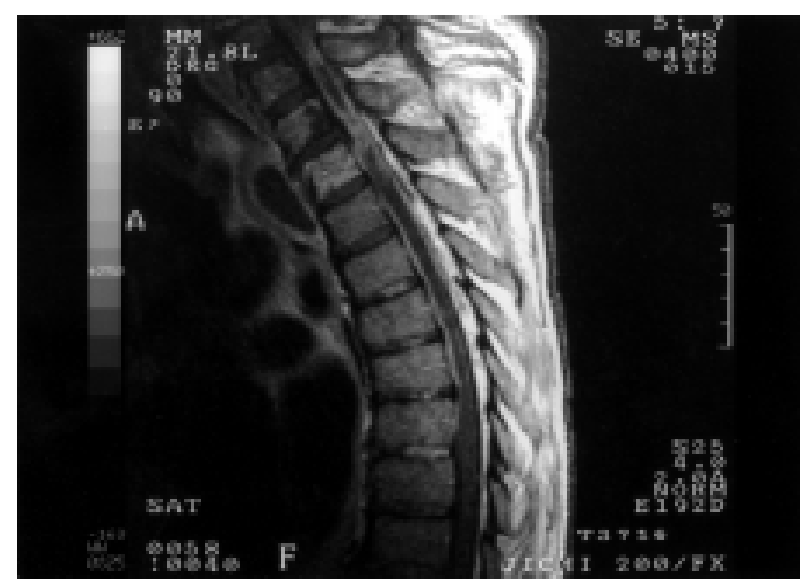

Figure 3. Computed transitional tomography scan of the pelvis in the second case. Abscess formations with a gas component are obvious in the left side of the retroperitoneal space.

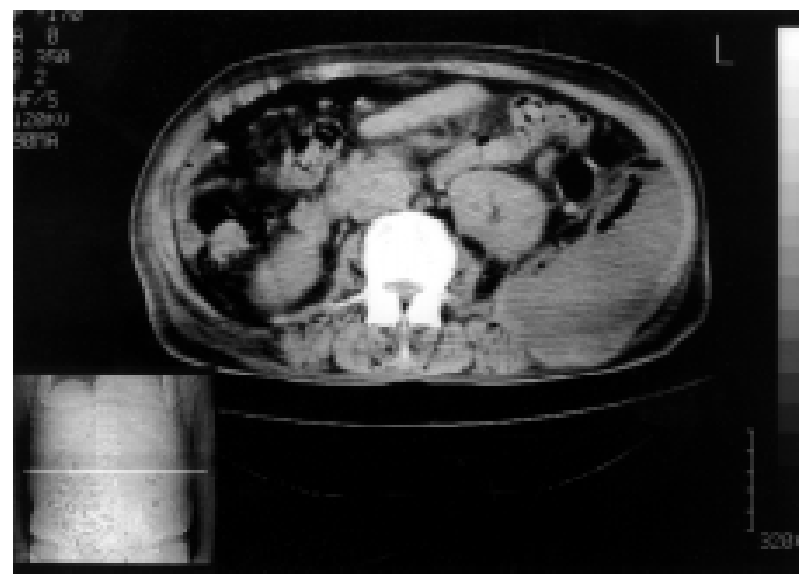


resolution of multiple abscesses. In contrast, paraplegia associated with the spinal epidural abscess did not improve with such treatments. In this case, late diagnosis and treatment for the retroperitoneal abscess induced coma, which made it difficult to diagnose the spinal epidural abscess. Leys and co-workers have proposed non-surgical treatment of spinal epidural abscesses [11].

In conclusion, we treated two diabetic patients with severe low back pain due to retroperitoneal abscess formation. Although multiple abscess formation, including a spinal epidural abscess, disappeared with intensive care, paraplegia was not relieved in case 1. Prompt diagnosis and treatment are necessary for this condition. We would like to advise that the possibility of abscess formation such as retroperitoneal abscess should be considered if a diabetic patient with fever complains of low back pain.

\section{Acknowledgement}

The authors thank Dr. Toshihide Ohmori for his critical reading of this manuscript and Ms. Takami Ohmori for her arrangement of the manuscript.

\section{References}

1. Lipsky B.A., Pecoraro R.E., Chen M.S., et al. Factors affecting staphylococcal colonization among NIDDM outpatients. Diabetes Care 1987;10:483-6.

2. Felig P., Baxter J.D., Frohman L.A. Endocrinology and Metabolism. McGraw-Hill, 1955.

3. Treiman G.S., Treiman R.L., Foran R.F., et al. The influence of diabetes mellitus on the risk of abdominal aortic surgery. Am Surg 1994;6:436-40.

4. Maslen D.R., Jones S.R., Crislip M.A., et al. Spinal epidural abscess-Optimizing patient care. Arch Intern Med 1993: 153;713-22.

5. Baker A.S., Ojemann R.G., Swartz M.N. Spinal epidural abscess. N Engl J Med 1975;293:463-8.

6. Danner R.L., Hartman B.J. Update of spinal epidural abscess: 35 cases and review of the literature. Rev Infect Dis 1987;9:265-74.

7. Knight J.W., Cordingley J.J., Palazzo G.A. Epidural abscess following epidural steroid and local anesthetic injection. Anaesthesia 1997;52:576-85.

8. Chao D., Nanda A. Spinal epidural abscess: a diagnostic challenge. Am Fam Physician 2002;65:1341-6.
9. Ohmori M., Harada K., Kitoh Y., et al. The functions of circulatory polymorphonuclear leukocytes in diabetic patients with and without diabetic triopathy. Life Sci 2000;66:1861-70.

10. Capecchi P.L., Blardi P., DeLalla A., et al. Pharmacokinetics and pharmacodynamics of neutrophil-associated ciprofloxacin in humans. Clin Pharmacol Ther 1995; 57:446-54.

11. Leys D., Lesoin F., Viaud C., et al. Decreased morbidity from acute bacterial spinal epidural abscess using computed tomography and nonsurgical treatment in selected patients. Ann Neurol 1985; 17:350-5. 